\title{
Entanglement teleportation via two-qubit Heisenberg interaction in Jaynes-Cummings model under intrinsic decoherence
}

\author{
A Naji, R Hamzehofi, and D Afshar \\ Department of Physics, Faculty of Science, Shahid Chamran University of Ahvaz, Ahvaz, Iran
}

(Received 12 March 2019 ; in final form 29 June 2019)

\begin{abstract}
In this paper, we investigate the entanglement teleportation of a two-qubit state under XYZ Heisenberg interaction in a cavity using the Jaynes-Cummings model. We analyze the channel entanglement, output entanglement and fidelity as a function of decoherence rate and spin coupling constant. We find that the teleportation is more effective if we take the proper initial input state.
\end{abstract}

Keywords: teleportation, entanglement, fidelity, decoherence, Jaynes-Cummings model

\section{Introduction}

The quantum teleportation was introduced by bennet [1] as a technique for moving quantum states via a quantum channel with the help of local operation and classical communication [1].Various systems have been used as the quantum channel in the teleportation process [2-9]. One of the systems that can be used for teleportation is cavity quantum electrodynamics [1012]. The Jaynes-Cummings model is a simple description of the interaction between two-level atoms and single mode radiations, which can be a good choice as the quantum channel [13]. On the other hand, the interaction between the system and environment causes decoherence. Intrinsic decoherence, as proposed by Milburn, is a model for the description of an open system based on [14]. This model is applied in the study of the decoherence effects in open quantum systems [15] and in the Jaynes-Cummings model [14].

In this article, we intend to study the teleportation via twoqubit Heisenberg interaction in the Jaynes-Cummins model and intrinsic decoherence. We have organized the paper as follows. In section 2, the Hamiltonian of the system is introduced and the density matrix of the system is obtained. In section 2, we study the entanglement of the quantum channel and the output state. Teleportation and fidelity properties are analyzed in sections 3 and 4 , respectively. Finally, in section 5, the conclusions is made.

\section{The Hamiltonian of the system}

The system consists of two two-level atoms with the Heisenberg interaction in a single-mode cavity. The Hamiltonian of the system can be written as [15].

$$
H=H_{\text {int }}+H_{\text {field }}+H_{\text {atoms }}
$$

where $H_{\text {int }}$ is the atom-cavity interaction Hamiltonian, $H_{\text {field }}$ is the field Hamiltonian and $H_{\text {atoms }}$ is the atoms Hamiltonian. With rotating wave approximation [15]

$$
\begin{aligned}
& H_{\text {int }}=\Omega\left(a \sigma_{1}^{+}+a \sigma_{2}^{+}+a^{\dagger} \sigma_{1}^{-}+a^{\dagger} \sigma_{2}^{-}\right), \\
& H_{\text {field }}=v a^{\dagger} a, \\
& \left.H_{\text {atoms }}=\frac{\omega}{2}\left(\sigma_{1}^{z}+\sigma_{2}^{z}\right)+J_{x} \sigma_{1}^{x} \sigma_{2}^{x}+J_{y} \sigma_{1}^{y} \sigma_{2}^{y}+J_{z} \sigma_{1}^{z} \sigma_{2}^{z}\right),
\end{aligned}
$$

where $\Omega$ is the atoms-field coupling coefficient. We suppose atoms and field have equal coupling coefficients. $J_{i}(i=x, y, z)$ is the spin coupling constant, $\omega$ is the tradition frequency between two states of the atoms, $v$ is the frequency of the field, and $a$ and $a^{\dagger}$ are the annihilation and creation operators respectively; $\sigma_{i}^{x, y, z}(i=1,2)$ refers to the Pauli matrices and $\sigma_{i}^{ \pm}=\frac{1}{2}\left(\sigma_{i}^{x} \pm \sigma_{i}^{y}\right)$. Also, we suppose the atoms and field are resonant; then $v=\omega$.

The Hamiltonian can be written as

$$
\begin{aligned}
H= & -J_{z}|\uparrow \downarrow 0\rangle\langle\uparrow \downarrow 0|+J| \downarrow \uparrow 0\rangle\langle\uparrow \downarrow 0|+J| \uparrow \downarrow 0\rangle\langle\downarrow \uparrow 0| \\
& -J_{z}|\downarrow \uparrow 0\rangle\langle\downarrow \uparrow 0|+\Omega| \downarrow \downarrow 1\rangle\langle\uparrow \downarrow 0|+\Omega| \downarrow \downarrow 1\rangle\langle\downarrow \uparrow 0| \\
& +\Omega|\downarrow \uparrow 0\rangle\rangle\left\langle\downarrow \downarrow 1\left|+J_{z}\right| \downarrow \downarrow 1\right\rangle\langle\downarrow \downarrow 1|+\Omega| \uparrow \downarrow 0\rangle\langle\downarrow \downarrow 1|,
\end{aligned}
$$

where $J=J_{x}+J_{y} . \uparrow$ means that atom is in the excited state, $\downarrow$ 


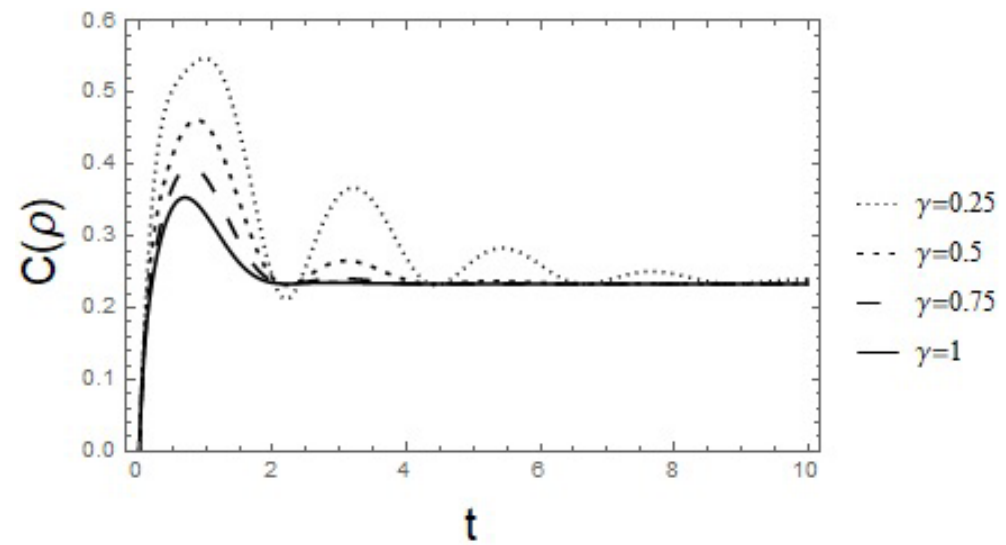

Figure 1. Concurrence of two atoms with $\Omega=1, J_{x}=0.5, J_{y}=0.75$, and $J_{z}=1$.

shows that atom is in the ground state, $|0\rangle$ represents that field is in the vacuum state and $|1\rangle$ means field is in the excited state. The matrix representation of the Hamiltonian can be written as:

$$
H=\left[\begin{array}{cccccccc}
0 & 0 & 0 & 0 & 0 & 0 & 0 & 0 \\
0 & -J_{z} & J & 0 & 0 & 0 & 0 & \Omega \\
0 & J & -J_{z} & 0 & 0 & 0 & 0 & \Omega \\
0 & 0 & 0 & 0 & 0 & 0 & 0 & 0 \\
0 & 0 & 0 & 0 & 0 & 0 & 0 & 0 \\
0 & 0 & 0 & 0 & 0 & 0 & 0 & 0 \\
0 & 0 & 0 & 0 & 0 & 0 & 0 & 0 \\
0 & \Omega & \Omega & 0 & 0 & 0 & 0 & J_{z}
\end{array}\right],
$$

The Non-zero Eigenvalues and the corresponding eigenvectors of $\mathrm{H}$ are given by

$E_{1}=-J-J_{z}$,

$E_{2}=\frac{1}{2}(J-A)$

$E_{3}=\frac{1}{2}(J+A)$,

$\left|\psi_{1}\right\rangle=-|\uparrow \downarrow 0\rangle+|\downarrow \uparrow 0\rangle$,

$\left|\psi_{2}\right\rangle=\frac{B-2 J_{z}}{4 \Omega}|\uparrow \downarrow 0\rangle+\frac{B-2 J_{z}}{4 \Omega}|\downarrow \uparrow 0\rangle+|\downarrow \downarrow 1\rangle$,

$\left|\psi_{3}\right\rangle=\frac{C-2 J_{z}}{4 \Omega}|\uparrow \downarrow 0\rangle+\frac{C-2 J_{z}}{4 \Omega}|\downarrow \uparrow 0\rangle+|\downarrow \downarrow 1\rangle$,

where $A=\sqrt{8 \Omega^{2}+\left(J-2 J_{z}\right)^{2}}, B=J-\sqrt{8 \Omega^{2}+\left(J-2 J_{z}\right)^{2}}$,

$C=J+\sqrt{8 \Omega^{2}+\left(J-2 J_{z}\right)^{2}}$.

The master equation describing the intrinsic decoherence is given by [16]

$$
\frac{d \rho(t)}{d t}=\gamma\left[e^{(-i H / \gamma)} \rho(t) e^{(-i H / \gamma)}-\rho(t)\right]
$$

where $\gamma$ is the intrinsic decoherence rate. Dynamics of the density operator $\rho(t)$ with the initial state $\rho(0)$ is given by [17].

$$
\begin{aligned}
\rho(t)=\sum_{m, n} \exp [ & \left.-\frac{\gamma}{2}\left(E_{m}-E_{n}\right)^{2}-i\left(E_{m}-E_{n}\right) t\right] \\
& \times\left\langle\psi_{m}|\rho(0)| \psi_{n}\right\rangle\left|\psi_{m}\right\rangle\left\langle\psi_{n}\right|,
\end{aligned}
$$

where $E_{i}(i=m, n)$ and $\psi_{i}(i=m, n)$ are the eigenvalues and the corresponding eigenvectors of $\mathrm{H}$, as given in the equation (7). We consider that the system is initially in the $|\uparrow \downarrow 0\rangle$ state. So, $\rho(0)$ reads:

$$
\rho(0)=|\uparrow \downarrow 0\rangle\langle\uparrow \downarrow 0|,
$$

With substituting (7) into equation (9), we can obtain the density matrix of the whole system; after tracing out the degree of the field, the reduced density matrix can read with the following form:

$$
\rho(t)=\left[\begin{array}{llll}
0 & 0 & 0 & 0 \\
0 & A & B & 0 \\
0 & C & D & 0 \\
0 & 0 & 0 & E
\end{array}\right],
$$

The density matrix of the system is too complicated to write out here, so we will discuss the numerical results.

\section{Entanglement of channel}

The concurrence of two qubits is defined by [18]

$C(\rho)=\max \left(0, \lambda_{1}-\lambda_{2}-\lambda_{3}-\lambda_{4}\right)$,

where $\lambda_{i}(i=1,2,3,4)$ refers to the square roots of the eigenvalues of the density matrix in a decreasing order

$$
R=\rho\left(\sigma_{y} \otimes \sigma_{y}\right) \rho^{*}\left(\sigma_{y} \otimes \sigma_{y}\right),
$$

where $\sigma_{y}$ is one of the Pauli matrices. The concurrence for $\rho(t)$ in the equation (11) is a function of the system parameters. Numerical simulation results for this case are shown in figure 1 . We have plotted the curves for four values of $\gamma$ by considering $0 \leq \gamma \leq 1$.

It is observed that the entanglement shows an oscillating behavior in time. Its amplitude is a decreasing function of $\gamma$; this is because by increasing the intrinsic decoherence, the chaos in the system is increased. Also, with the passage of time, the concurrence approaches a stable value. It means even under high intrinsic decoherence, the quantum channel remains entangled.

\section{Entanglement Teleportation}

According to the standard teleportation, we consider Alice and Bob who share an entangled state given by equation (11). Alice is given the pure state $\rho_{\text {in }}=\left|\psi_{\text {in }}\right\rangle\left\langle\psi_{\text {in }}\right|$, where $\left|\psi_{\text {in }}\right\rangle=\cos \frac{\theta}{2}|10\rangle+e^{i \varphi} \sin \frac{\theta}{2}|01\rangle$ and $(0 \leq \theta \leq \pi, 0 \leq \varphi \leq 2 \pi)$.

The aim of Alice is to send this state by equation (11). The output state is given by [17]

$\rho_{\text {out }}=\sum_{\mu v} p_{\mu v}\left(\sigma_{\mu} \otimes \sigma_{v}\right) \rho_{\text {in }}\left(\sigma_{\mu} \otimes \sigma_{v}\right)$,

where $\sigma_{\mu, v}(\mu, v=1,2,3)$ refers to the Pauli matrices and 


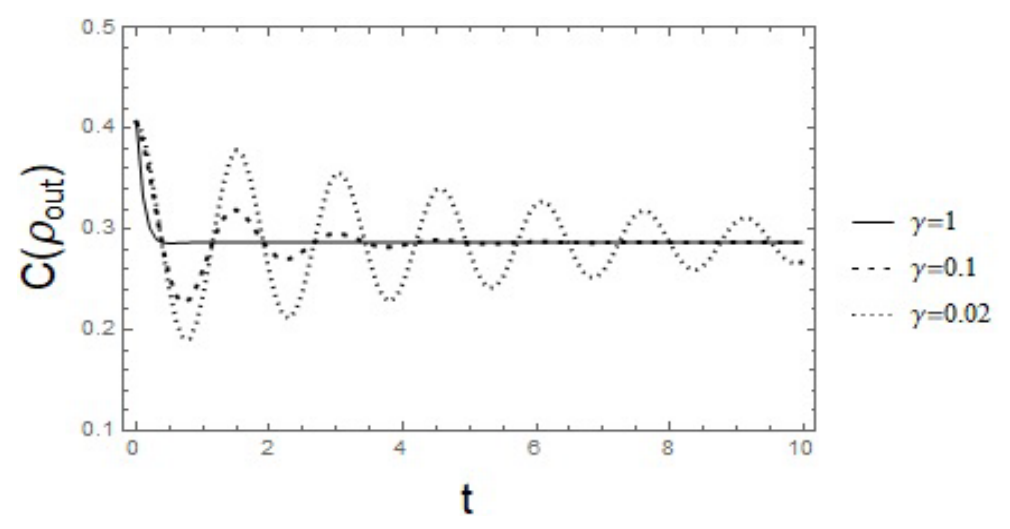

Figure 2. Concurrence of the output state with $\Omega=1, J_{x}=1, J_{y}=2, J_{z}=3$, and $\varphi=0, \theta=\frac{\pi}{2}$.

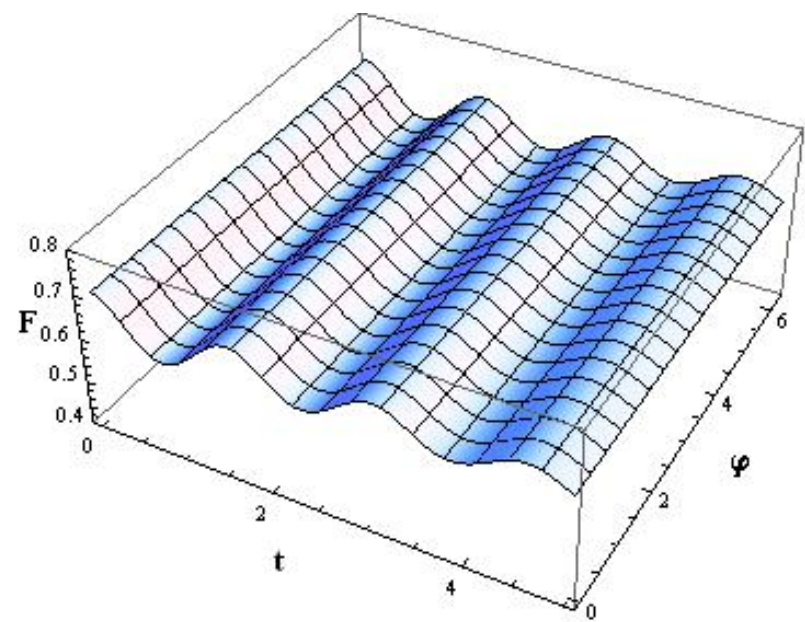

Figure 3. (color online) Fidelity of system versus $t-\varphi$ with $\gamma=0.02, \Omega=1, J_{x}=1, J_{y}=2, J_{z}=3$, and $\theta=\pi$.

$p_{\mu \nu}=\operatorname{Tr}\left[E^{\mu} \rho(t)\right] \operatorname{Tr}\left[E^{v} \rho(t)\right]$,

$\sum_{\mu v} p_{\mu v}=1$,

$E^{0}=\left|\psi^{-}\right\rangle\left\langle\psi^{-}\right|$,

$E^{1}=\left|\phi^{-}\right\rangle\left\langle\phi^{-}\right|$,

$E^{2}=\left|\phi^{+}\right\rangle\left\langle\phi^{+}\left|, E^{3}=\right| \psi^{+}\right\rangle\left\langle\psi^{+}\right|$,

$\phi^{ \pm}=(1 / \sqrt{2})(|00\rangle \pm|11\rangle) \psi^{ \pm}=(1 / \sqrt{2})(|01\rangle \pm|10\rangle)$.

We calculated the entanglement for the output state $\rho_{\text {out }}$. The result is shown in figure 2 . It can be observed that at $t=0$, the entanglement begins around 0.4 and it is an oscillating function of time; also, the amplitude of the entanglement is a decreasing function of the decoherence parameter $\gamma$. Further, as time passes, the concurrence keeps a stable value.

\section{Dynamics of fidelity}

The quality of the teleported state will be measured in terms of fidelity. The fidelity of the two states is given by [19]

$$
F\left(\rho_{\text {in }}, \rho_{\text {out }}\right)=\left\{\operatorname{Tr}\left[\sqrt{\sqrt{\rho_{\text {in }}} \rho_{\text {out }} \sqrt{\rho_{\text {in }}}}\right]\right\}^{2},
$$

where $\rho_{\text {in }}$ is the input state of the channel and $\rho_{\text {out }}$ is the output state of the channel.

We have presented the plots of the fidelity as a function of time and $\varphi$ in figure 3 and also, as function time and $\theta$ in

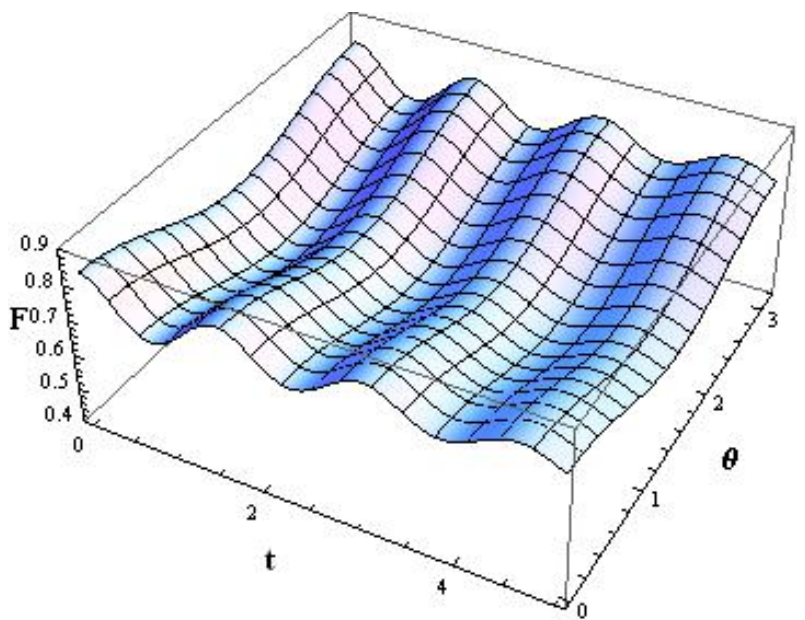

Figure 4. (color online) Fidelity of system versus $t-\theta$ with $\gamma=0.02, \Omega=1, J_{x}=1, J_{y}=2, J_{z}=3$, and $\varphi=0$.

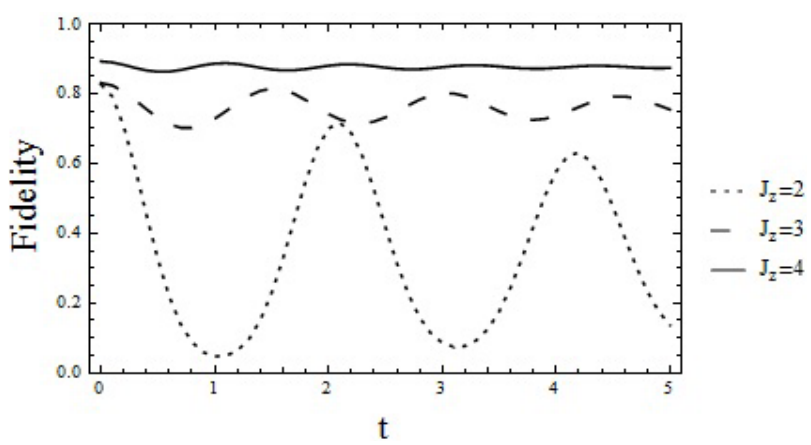

Figure 5. Fidelity versus time with $\gamma=0.02, \Omega=1, J_{x}=1$, $J_{y}=2, \varphi=0$, and $\theta=0$.

figure 4. According to the plots in figure 3, we note that the fidelity is independent of $\varphi$. Also, figure 4 shows that the fidelity becomes maximum at $\theta=0, \theta=\pi$ and minimum at $\theta=\pi / 2$. Moreover, the fidelity shows the oscillatory behavior. It is observed that the fidelity is optimum for the input state with the values $\theta=0$ or $\theta=\pi$. Therefore, we use this optimal input state in the next plots.

To see the role of the spin coupling constant, we have depicted the fidelity as function of time for three values of $J_{i}(i=x, y, z)$ in figures 5,6 , and 7 . This implies that the fidelity is increased by increasing one of the spin coupling constants. This is because by increasing the spin coupling constant, the entanglement of the channel is increased too, 


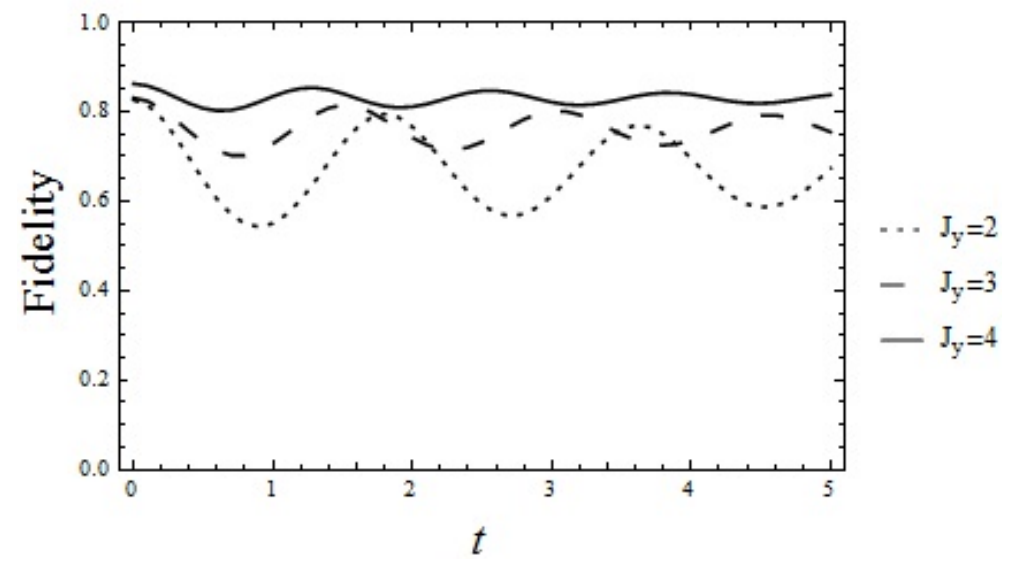

Figure 6. Fidelity versus time with $\gamma=0.02, \Omega=1, J_{x}=2, J_{z}=1, \varphi=0$, and $\theta=0$.

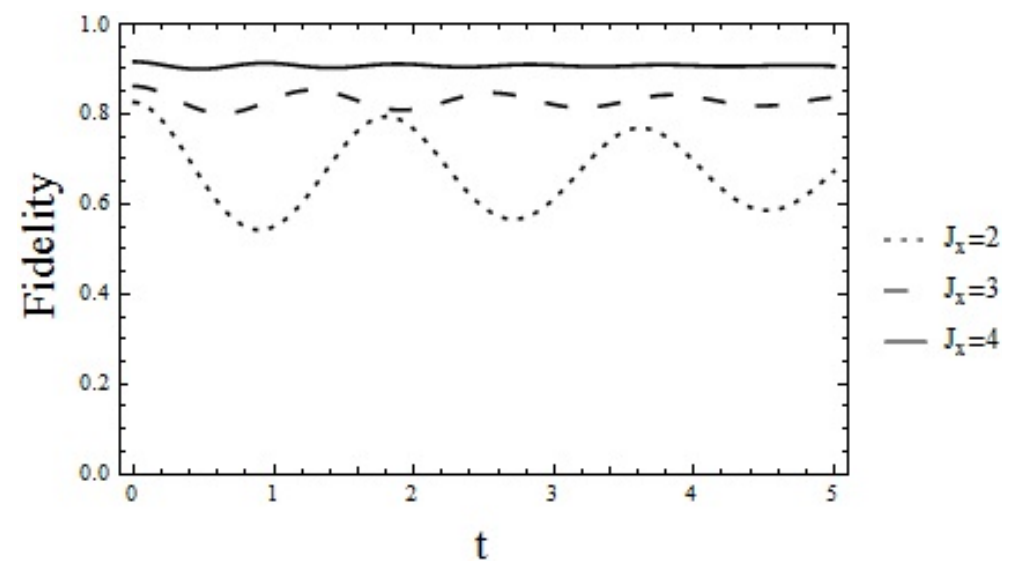

Figure 7. Fidelity versus time with $\gamma=0.02, \Omega=1, J_{y}=2, J_{z}=1, \varphi=0$, and $\theta=0$.

optimizing the entanglement teleportation.

\section{Conclusions}

We have studied the dynamics of entanglement and the fidelity of the teleportation in a two-qubit state under XYZ Heisenberg interaction coupled to a cavity in the Jaynes-Cummings model under intrinsic decoherence. A summary of the important results is as follows:

\section{References}

1.C H Bennett, G Brassard, C Crepeau, R Jozsa, A Peres, and W K Wotters, Phys. Rev. Lett. 70 (1997) 1895.

2. M Riebe, M Chwalla, J Benhelm, H Haffner, W Hansel, C F Roos, and R Blatt, New Journal of Phys. 9 (2007) 211.

3. X Xu, and $\mathrm{X}$ wang, International Journal of Theoretical Phys. 55 (2016) 3551.

4. J Joo and E Ginossar, Nature 6 (2016) 26338.

5. G F Zhang, Physical Review. A. 75 (2007) 1050.

6. H Mohammadpour and M Zareyan, Iranian J. Phys. Res. 8, 2 (2008) 114



$$
\begin{aligned}
& .11 \%(1 \mathrm{HV})
\end{aligned}
$$

7.H Mohammadi, S J Akhtarshenas, and F Kheirandish, Eur. Phys. J. D 62 (2011) 439.

8. M Huo, J Qin, J Cheng, Z Yan, Z Qin, X Su, X Jia, C Xie, and K Peng, Science Advances 4, 10 (2018) eaas9401.

9. X Tan, X Li, and $\mathrm{P}$ Yang, Computers, Materials and Continua 57 (2018) 495.
The quantum channals is always entangled. The concurrence oscillates in times and its amplitude is decreased by the decoherence rate and time. The output state is always entangled and the concurrence of the output state is less than that of the channel all times. The fidelity is maximum if we choose $\theta=0$, $\theta=\pi$ for the input state. The fidelity is increased by increasing one of the components of the spin coupling constant.

10. N Metwally, M Abdelaty, and A S F Obada, Chaos, Solitons \& Fractals 22 (2004) 529.

11. A Smirne, D Brivio, S Cialdi, B Vacchini, and M G A Paris, Phys. Rev. A 84 (2011) 032112.

12. F Mirmasoudi and S Ahadpour, Journal of Modern Optics 65 (2017) 730.

13. E T Jaynes and F W Cummings, "Comparison of Quantum and Semiclassical Radiation Theories With Application to the Beam Maser", Proc. IEEE. 51 (1963) 89.

14. G J Milburn, Phys. Rev. 44 (1991) 5401.

15. P Lambropoulos and D Petrosyan, "Fundamentals of Quantum Optics and Quantum Information", Springer (2007).

16. L Zheng and G Zhang, Eur. Phys. J. D 71 (2017) 288.

17. J L Guo and H S Song, Phys. Scripta 78 (2008) 2055.

18. D McMahon, "Quantum Computing Explained", Wiley (2007).

19.R Jozsa, Journal of Modern Optics 41 (1994) 2315. 